\title{
TEMPO DESPENDIDO NA EXECUÇÃO DO PROCESSO DE ENFERMAGEM EM UM CENTRO DE TRATAMENTO INTENSIVO
}

\author{
Time spent on carrying out the nursing process in an intensive care unit \\ Tiempo en la aplicación del proceso de enfermería en una unidad de cuidados \\ intensivos \\ Miriam de Abreu Almeida ${ }^{1}$ \\ Isis Marques Severo² \\ Enaura Brandão Chaves ${ }^{3}$ \\ Luciana Nabinger Menna Barreto ${ }^{4}$ \\ Daniela Marona Borba ${ }^{5}$
}

\section{RESUMO}

Este estudo objetivou mensurar o tempo despendido pelos enfermeiros de um Centro de Terapia Intensiva (CTI) na execução das etapas do Processo de Enfermagem (PE). Trata-se de uma investigação descritiva, observacional e exploratória, com metodologia quantitativa. Foram cronometradas as etapas do PE realizadas por seis enfermeiros em 29 pacientes internados no CTI. A pesquisa foi aprovada pelo Comitê de Ética em Pesquisa da instituição. 0 tempo foi mensurado nas etapas: avaliação diária do paciente: 8,34 $(3,78)$; diagnóstico e prescrição de enfermagem: 3,65 (2,27-5,45); aprazamento da prescrição de enfermagem: 2,30 (1,14); e evolução da assistência de enfermagem: 11,29 (2,55). 0 enfermeiro despendeu 25,58 minutos por paciente na realização do PE. Os dados fornecem subsídios para o dimensionamento de recursos humanos no cuidado a pacientes críticos, com vistas à qualificação da assistência.

Palavras-chave: Processos de enfermagem. Unidades de terapia intensiva. Diagnóstico de enfermagem.

\begin{abstract}
This study had as a goal the measure of time spent on an Intensive Care Unit (ICU) by nurses when carrying out the steps of the Nursing Process (NP). It is a descriptive research, observational and exploratory, using quantitative methodology. Steps of the NP as carried out were timed by six nurses on 29 ICU patients. Research was approved by the Institutional Ethics Review Board. Time was measured in the following steps: daily patient assessment: 8.34 (3.78); diagnosis and nursing prescription: 3.65 (2.27-5.45); scheduling nursing prescription: 2.30 (1.14) and evaluation of care: 11.29 (2.55). Nurses spent 25.58 minutes per patient when carrying out the NP. The data provide a way to measure human resources required for caring for critical patients, so as to qualify care giving.
\end{abstract}

Keywords: Nursing process. Intensive care units. Nursing diagnosis.
Resumen

Este estudio tuvo como objetivo medir el tiempo dedicado por el personal de enfermería en una Unidad de Cuidados Intensivos (UCl) en la aplicación de los pasos del Proceso de Enfermería (PE). Se trata de una investigación descriptiva, observacional y exploratoria, utilizando el método cuantitativo. Fueron cronometradas las etapas del PE llevadas a cabo por seis enfermeros en 29 pacientes internados en la UCl. El estudio fue aprobado por la Comisión de Ética en Investigación de la Institución. Se midió el tiempo en cada fase: evaluación diaria del paciente: 8,34 (3,78); diagnóstico y la prescripción de enfermería: 3.65 (2.27-5.45); programación de la prescripción en enfermería: 2.30 (1, 14); y la evolución de asistencia: 11,29 (2,55). El enfermero pasó 25.58 minutos por paciente en la realización del PE. Los datos fueron importantes para dimensionar los recursos humanos necesarios para el cuidado de pacientes críticos, con el fin de calificar la asistencia.

Palabras clave: Procesos de enfermería. Unidades de terapia intensiva. Diagnóstico de enfermería.

${ }^{1}$ Enfermeira, Doutora em Educação pela PUCRS. Professora Associada do Departamento de Enfermagem Médico-Cirúrgica da Universidade Federal do Rio Grande do Sul (UFRGS). Coordenadora da Comissão do Processo de Enfermagem do Hospital de Clínicas de Porto Alegre (HCPA). Porto Alegre - RS. Brasil. E-mail: miriam.abreu2@gmail.com; ²Doutoranda em Enfermagem - Universidade Federal do Rio Grande do Sul (UFRGS). Preceptora da Residência Integrada Multiprofissional em Saúde (RIMS) do HCPA - Ênfase Adulto Crítico. Porto Alegre - RS. Brasil. E-mail: isis_severo@hotmail.com; ${ }^{3}$ Doutora em Enfermagem pela UNIFESP. Mestre em Administração pela UFRGS. Professora Assistente do Departamento de Orientação e Administração Profissional da Escola de Enfermagem da UFRGS. Chefe do Serviço de Enfermagem em Terapia Intensiva do HCPA. Porto Alegre - RS. Brasil. E-mail: enaurah@hotmail.com; ${ }^{4}$ Mestranda em Enfermagem pela UFRGS. Enfermeira do Hospital Banco de Olhos. Porto Alegre - RS. Brasil. E-mail: luciana.nabinger@gmail.com; ${ }^{5}$ Mestre em Enfermagem pela UFRGS. Enfermeira Chefe da Unidade de Terapia Intensiva do HCPA. Porto Alegre - RS. Brasil. E-mail: danimarona@hotmail.com 


\section{INTRODUÇÃO}

0 Processo de Enfermagem (PE) é a estrutura conceitual sólida para a prestação de cuidados, garantindo sua sistematização e continuidade. É a partir dela que a profissão atingirá sua maioridade, porém a autonomia desejada por todos os profissionais enfermeiros só será alcançada no momento em que toda a classe passar a utilizar essa metodologia científica em suas ações ${ }^{1}$.

0 Processo de Enfermagem é um modelo metodológico ou instrumento tecnológico do enfermeiro que favorece o cuidado, organizando as condições necessárias à realização do cuidado e documentando a prática profissional. Este modelo contribui para visibilidade e reconhecimento social e representa uma possibilidade de avaliação da prática do profissional².

Trata-se de um método que começou a ser divulgado no Brasil no início dos anos 70 e que se desenvolve em seis etapas: a primeira é a coleta de dados (Histórico de Enfermagem), considerada fundamental para subsidiar a segunda fase, que corresponde ao Diagnóstico de Enfermagem, em que o enfermeiro analisa e julga os dados coletados, identifica os problemas de enfermagem, estabelece as necessidades básicas afetadas e determina o grau de dependência do paciente. A terceira fase é o plano assistencial; a quarta fase corresponde à prescrição das intervenções de enfermagem; a quinta é a avaliação (evolução diária); e a sexta, e última etapa, denominada prognóstico de enfermagem, é a estimativa da capacidade do indivíduo em atender suas próprias necessidades básicas alteradas, após a implementação do plano de cuidados'.

No entanto, é preciso que o PE seja aderido pela equipe de enfermagem e sua utilização seja sistematizada para que seu uso realmente aperfeiçoe e qualifique o cuidado prestado ao paciente. Desta forma, a Sistematização da Assistência de Enfermagem (SAE) e o Processo de Enfermagem precisam andar lado a lado para que se obtenham os melhores resultados para o paciente, para o profissional de enfermagem e para a instituição.

A implementação da SAE constitui uma exigência para as instituições de saúde públicas e privadas de todo 0 Brasil, de acordo com a resolução do Conselho Federal de Enfermagem (COFEN) n $n^{0}$ 358/20093. Trata-se também de uma orientação da lei do exercício profissional da Enfermagem ${ }^{4}$ (Lei 7.498, de 25 de junho de 1986). Além disso, sua implementação se configura em uma estratégia na organização da assistência de enfermagem nas instituições, atendendo, assim, aos requisitos do Manual Brasileiro da Acreditação Hospitalar ${ }^{5}$.

A equipe de enfermagem do Hospital de Clínicas de Porto Alegre (HCPA) vem trabalhando com a metodologia do $P E$ no cuidado prestado aos pacientes há mais de duas décadas. A SAE partiu da preocupação do grupo de professores e enfermeiros que ali atuam na área de ensino ou no campo prático, com o desenvolvimento de cuidados cada vez mais seguros e aprimorados aos pacientes, bem como a visibilidade social da profissão.

Esta sistemática vem sendo continuamente aprimorada e conta atualmente com uma Comissão do Processo de Enfermagem (COPE) composta por docentes e enfermeiros representantes de todas as áreas assistenciais da instituição que se dedicam ao estudo e operacionalização do PE no HCPA.

$0 \mathrm{PE}$ incorporado à prática de enfermagem na instituição vive hoje o momento de informatização em quase todas as áreas em uma implantação gerenciada de forma gradativa e organizada. Ela tem sido subsidiada por levantamentos e pesquisas ${ }^{6-9}$ que são realizadas pelas áreas específicas direcionadas a certas etapas do processo ou abrangendo-o como um todo.

0 Centro de Tratamento Intensivo (CTI) do HCPA é uma das áreas assistenciais comprometidas com 0 desenvolvimento do PE dentro da instituição, e os enfermeiros desenvolvem todas as suas etapas, estabelecendo os diagnósticos de enfermagem e a prescrição informatizada dos pacientes pelos quais são responsáveis durante seus turnos de trabalho. Tendo em vista a dinâmica do trabalho da equipe de enfermagem no cuidado aos pacientes críticos, e a relevância da metodologia do PE na sistematização do mesmo, considerou-se importante realizar uma avaliação do tempo despendido pelo enfermeiro no desempenho dessas atividades, que segundo a lei que rege seu exercício profissional constitui-se uma função de sua exclusiva responsabilidade 4 .

Assim sendo, o objetivo deste estudo foi mensurar o tempo despendido pelos enfermeiros de um Centro de Tratamento Intensivo na execução das etapas do Processo de Enfermagem.

\section{METODOLOGIA}

Estudo observacional, exploratório e descritivo com metodologia quantitativa ${ }^{10-11}$. Faz parte de um projeto maior intitulado "Mensuração do tempo despendido pelos enfermeiros de um hospital universitário na Sistematização da Assistência de Enfermagem"12, aprovado pela Comissão de Pesquisa da Escola de Enfermagem da Universidade Federal do Rio Grande do Sul (EEUFRGS) - COMPESQ e pelo Comitê de Ética em Pesquisa do HCPA sob o número 07-598.

Foi desenvolvido no CTI adulto do HCPA, Rio Grande do Sul, onde o PE consiste nas etapas: anamnese e exame físico, diagnóstico de enfermagem (DE), e prescrição e evolução da assistência de enfermagem; a primeira etapa tem diferentes modelos de acordo com as especificidades de cada área, norteados pelo referencial teórico das necessidades humanas básicas de Wanda Aguiar Horta'.

Na unidade estudada desde fevereiro de 2010 todas as etapas do PE são informatizadas; no entanto, até janeiro do mesmo ano, a evolução da assistência e o instrumento que 
serve de registro da anamnese e exame físico eram documentados manualmente.

0 CTI contém 39 leitos de internação que são referência para pacientes clínicos e cirúrgicos de diversas especialidades, como neurologia, pneumologia, nefrologia, ginecologia, entre outros. A equipe de enfermagem constitui-se de 53 enfermeiros e 140 técnicos de enfermagem diretamente ligados à assistência do paciente.

A amostra desta pesquisa foi constituída por seis enfermeiros que atuam no CTI. A seleção foi intencional mediante convite.

Os critérios de inclusão foram: ter experiência de 2 anos ou mais na realização do $\mathrm{PE}$, ter recebido capacitação sobre o processo de enfermagem ao ser admitido no HCPA e ser egresso da Escola de Enfermagem da Universidade Federal do Rio Grande do Sul (UFRGS), onde o DE é ensinado em disciplinas que compõem o currículo, uma vez que executam o $P E$ nas unidades de internação da instituição, que é campo de prática específico. Ao ingressarem no HCPA, os enfermeiros recebem capacitação sobre o PE.

Os critérios de exclusão foram: ser chefe de unidade e/ ou supervisor de estágio, pelo fato de estes enfermeiros estarem envolvidos em supervisão e ensino.

0 cálculo da amostra de pacientes foi realizado maximizando a variância, ou seja, assumindo uma proporção de 50\%, visto que não existem informações prévias a respeito destas estimativas. Para o cálculo foi considerada uma confiança de $95 \%$ e um erro máximo da estimativa de $10 \%$. Com base no número de internações no período de janeiro a abril de 2007 ( $n=40$ ), foi realizada a correção para populações finitas, obtendo-se, assim, o tamanho final necessário de 29 pacientes na unidade estudada.

Os participantes assinaram o Termo de Consentimento Livre e Esclarecido (TCLE) seguindo as exigências da Resolução do Conselho Nacional de Saúde (CNS) 196/96 ${ }^{13}$.

A coleta dos dados foi conduzida pelos pesquisadores e se constituiu de observação direta das atividades relacionadas ao PE. Foram cronometradas as avaliações diárias, os DE, as prescrições de enfermagem e as evoluções dos pacientes realizadas no período de fevereiro a junho de 2008. Destaca- se que, no HCPA, a primeira etapa do PE (histórico de enfermagem ou coleta de dados) é denominada anamnese e exame físico, realizada na admissão, preferencialmente nas primeiras 24 horas de internação, de forma completa e abrangente, buscando a história clínica do paciente. Porém, diariamente todos os pacientes são avaliados pelo enfermeiro. $\mathrm{Na}$ avaliação diária também são realizados a anamnese e o exame físico, porém de forma sucinta, mas minuciosa, não sendo necessário resgatar a história clínica pregressa do paciente, já realizado no momento da internação. No sistema informatizado de prescrição do HCPA, o enfermeiro elabora os DE, segundo a NANDA- $1^{14}$ e seleciona as intervenções simultaneamente. Portanto, os DE e a prescrição de enfermagem são etapas que estão interligadas. As intervenções são cadastradas no sistema de acordo com a classificação da NIC ${ }^{15}$ e, também, a partir da prática clínica dos enfermeiros da instituição.

As observações foram feitas nos turnos da manhã e da tarde e realizadas pelos pesquisadores, os quais mensuraram 0 tempo de cada etapa do PE por meio de cronômetro. A mensuração iniciava quando o enfermeiro começava cada etapa até o seu término, parando o cronômetro quando era interrompido. Quando havia interrupção era registrado seu horário de início e término. Os dados provenientes da observação foram registrados em um roteiro de coleta de dados elaborado pelas autoras. Nele constaram a data da observação, o turno, o local, o nome do pesquisador, as atividades observadas e seus tempos mensurados, e, havendo interrupção, seu horário de início e fim.

\section{RESULTADOS}

Os resultados são apresentados no Quadro 1: dados com distribuição simétrica em tempo (minutos) e média (desviopadrão); dados com distribuição assimétrica em tempo (minutos) e mediana (25\%-75\%).

No período de coleta de dados não houve necessidade de realizar e documentar a etapa de anamnese e exame físico (histórico de enfermagem), porque os pacientes da amostra foram transferidos de outras unidades e já tinham esse registro. Se isso tivesse ocorrido, a média de tempo total do processo de enfermagem seria superior a 25,58 minutos por paciente, que correspondeu ao período de observação.

\begin{tabular}{ll}
\hline \multicolumn{1}{c|}{ ETAPAS DO PROCESSO DE ENFERMAGEM } & $\begin{array}{l}\text { TEMPO(MIN) } \\
\text { MÉDIA(DESVIO-PADRÃO) } \\
\text { MEDIANA(25\%-75\%) }\end{array}$ \\
\hline $\begin{array}{l}\text { Anamnese e exame físico (Histórico de } \\
\text { Enfermagem) }\end{array}$ & \\
Avaliação diária do paciente * & $8,34(3,78)$ \\
DE $^{14}$ e prescrição de cuidados (PC) ** & $3,65(2,27-5,45)$ \\
Aprazamento da PC * & $2,30(1,14)$ \\
Evolução da assistência de enfermagem * & $11,29(2,55)$ \\
\hline Total & 25,58 \\
\hline
\end{tabular}

Legenda: *distribuição simétrica, ** distribuição assimétrica 


\section{DISCUSSÃO}

0 Quadro 1 apresenta o tempo despendido e medido (em minutos) na realização das etapas do processo de enfermagem no CTI do HCPA. Na avaliação diária do paciente foi mensurado o tempo médio de 8,34 (desvio-padrão: 3,78) minutos.

0 tempo que o enfermeiro leva realizando o exame físico e o número de horas dos cuidados de enfermagem em unidade de terapia intensiva foi medido em estudo realizado em um hospital da rede privada de São Paulo. Nessa instituição foi mensurado o tempo de 12,33 minutos na realização do exame físico pelos enfermeiros ${ }^{16}$.

Já em um hospital da rede pública de São Paulo, o tempo despendido na unidade de terapia intensiva, no que se refere à avaliação do paciente, foi de 6,4 (desvio-padrão: 4,7) minutose, em seu seguimento, ou seja, em uma próxima avaliação, foi 4,1 (desvio-padrão: 2,1) minutos ${ }^{17}$. As autoras desse estudo comentam que o trabalho do enfermeiro em unidade de terapia intensiva, pela sua complexidade e precisão, exige dos seus executores o contato direto com situações de risco de morte e urgência. Essas situações inerentes ao trabalho nesse contexto podem influenciar o seu desenvolvimento e execução.

0 perfil do trabalho do enfermeiro em terapia intensiva exige sua capacidade de avaliação criteriosa, que necessita de habilidade para a realização da anamnese e exame físico e, consequentemente, raciocínio crítico das necessidades do paciente.

Os estudos do CTI do HCPA e de São Paulo não apresentaram, nesse momento, o tempo das interrupções do processo de enfermagem que acabam influenciando no seu desenrolar, pois muitas vezes, o enfermeiro precisa retomar todo o raciocínio diagnóstico, e nessa retomada leva mais tempo para sua execução.

OQuadro 1 apresenta também o tempo que o enfermeiro utiliza para realização da prescrição de enfermagem, mediana de 3,65 (2,27-5,45) minutos; em seu aprazamento, quando além de informatizado é complementado de forma manual, foi apresentada média de 2,30 (desvio-padrão: 1,14) minutos.

Rezende e Gaizinski ${ }^{17}$ identificaram o tempo para o registro da admissão, diagnósticos de enfermagem, prescrição e evolução do paciente na unidade de terapia intensiva de um hospital de São Paulo, chegando ao tempo de 17,4 minutos. As autoras não apresentam os dados de cada uma dessas etapas separadamente.

Outra pesquisa sobre a sistematização da assistência de enfermagem em terapia intensiva sustentada na teoria de Wanda Horta mostrou um tempo aproximado de 40 minutos para a realização do PE. Porém, durante o seu desenvolvimento, o enfermeiro recebia solicitações para a realização de procedimentos que retardavam a sua finalização. Nesta pesquisa não foi computado o tempo de cada uma das etapas do PE separadamente, nem o tempo das interrupções deste ${ }^{17}$.
No entanto, no período de coleta de dados, não houve necessidade de realizar e documentar a etapa de anamnese e exame físico (histórico de enfermagem), porque os pacientes da amostra tinham esse registro de outras unidades do hospital.

\section{CONSIDERAÇÕES FINAIS}

Os resultados da pesquisa proporcionam dados objetivos sobre o tempo despendido pelos enfermeiros do CTI do HCPA na execução do PE. Os dados com distribuição simétrica foram apresentados em tempo (minutos) e média (desvio-padrão); e os dados com distribuição assimétrica, em tempo (minutos) e mediana (25\%-75\%).

0 tempo do PE foi mensurado nas etapas: avaliação diária do paciente: 8,34 (3,78); diagnóstico e prescrição de enfermagem: 3,65 (2,27-5,45); aprazamento da prescrição de enfermagem: 2,30 (1,14); e evolução da assistência de enfermagem 11,29 (2,55).

Acreditamos que o fato de não termos despendido tempo na execução do histórico de enfermagem, no período da coleta, não se constitua em justificativa para a plena realização do PE no CTI, e sim reforçamos esse tempo na cuidadosa avaliação do paciente e revisão dos diagnósticos e intervenções de enfermagem.

Pretende-se com este estudo subsidiar o planejamento de recursos humanos visando a uma assistência qualificada, considerando que se somam ao tempo despendido no PE as interrupções ocorridas durante o período de trabalho, não especificadas nesse momento, tais como atender telefone, familiares e equipe, urgências com os demais pacientes, e ainda, resolver problemas de escalas, entre outros. Há intenção do grupo de dar continuidade ao estudo, repetindo a pesquisa após informatização do histórico de enfermagem e evolução de enfermagem, a fim de confrontar os achados.

\section{REFERÊNCIAS}

1. Horta WA. 0 processo de enfermagem. São Paulo: EPU; 1979.

2. Garcia TR, Nóbrega MML. Processo de enfermagem: da teoria à prática assistencial e de pesquisa. Esc Anna Nery. [periódico on-line]. 2009 mar [citado 2011 abr 27]; 13(1):816-18. Disponível em: http://www.scielo.br.

3.Conselho Federal de Enfermagem. Resolução COFEN 358/2009. Dispõe sobre a sistematização da assistência de enfermagem e a implantação do processo de enfermagem em ambientes públicos ou privados, em que ocorre o cuidado profissional de enfermagem, e dá outras providências. [citado 2010 set 12] Disponível em: http://www.portalcoren-rs.gov.br .

4 Lei $n^{0} 7.498$, de 25 de junho de 1986. Dispõe sobre a regulamentação do exercício da enfermagem e dá outras providências. Diário Oficial da República Federativa do Brasil, Brasília (DF), 26 jun 1986: Seção 1:1. 
Tempo despendido no processo de Enfermagem no CTI

5 Organização Nacional de Acreditação. Manual Brasileiro da Acreditação Hospitalar. $3^{\text {a }}$ ed. Brasília(DF): Ministério da Saúde; 2002.

6 Lucena AF. Mapeamento dos diagnósticos e intervenções de enfermagem em uma unidade de terapia intensiva. [tese de doutorado] São Paulo: Escola Paulista de Medicina/UFSP; 2006.

7 Franzen E, Almeida MA, Alitti G, Bercioni RR, Menegon DB, Rabelo ER. Adultos e idosos com doenças crônicas: implicações para o cuidado de enfermagem. Rev HCPA. 2007; 27(2): 28-31.

8 Moreira CLA. Atividades desenvolvidas pelos enfermeiros em unidade de internação pediátrica de um hospital-escola. [trabalho de conclusão]. Rio Grande do Sul: Escola de Enfermagem/UFRGS; 2007.

9 Almeida, MA, Aliti GB, Franzen E, Thomé EGR, Unicovsky MR, Rabelo ER, et al. Diagnóstico de enfermagem e intervenções prevalentes no cuidado ao idoso hospitalizado. Rev Lationo-Am Enfermagem. [periódico on-line]. 2008 jul-ago; [citado 2011 abr 22]; 16(4): [aprox. 6 telas]. Disponível em: http://www.scielo.br .

10 Goldim JR. Manual de iniciação à pesquisa em saúde. Porto Alegre: Dacasa; 2000.

11 Polit DF, Hungler B. Fundamentos de pesquisa em enfermagem. Porto Alegre: Artes Médicas; 1999.

12 Almeida MA, Magalhães AMM, Severo IMS, Dutra CF, Frare FF, Franzen E, et al. Mensuração do tempo despendido pelos enfermeiros de um hospital universitário na sistematização da assistência de enfermagem. [projeto de pesquisa]. Rio Grande do Sul: Universidade Federal do Rio Grande do Sul/UFRS; 2008.

13 Ministério da Saúde (BR). Conselho Nacional de Saúde. Resolução n ${ }^{\circ}$ 196, de 10 de outubro de 1996. Diretrizes e normas regulamentadoras de pesquisas envolvendo seres humanos. Brasília (DF); 1996.

14 NANDA International. Diagnósticos de enfermagem da NANDA: definições e classificação 2009-2011. Porto Alegre: Artmed; 2010.

15 Bulechek GM, Butcher HK, Dochterman JM. Classificação das Intervenções de Enfermagem (NIC). Rio de Janeiro: Elsevier; 2010.

16 Tranquitelli AM, Ciampone MHT. Número de horas de cuidados de enfermagem em unidade de terapia intensiva de adultos. Rev Esc Enferm USP. [periódico on-line]. 2007; [citado 2011 abr 22]; 41(3): [aprox. 7 telas]. Disponível em: http://www.scielo.br

17 Rezende PO, Gaizinski RR. Tempo despendido no sistema de assistência de enfermagem após implementação de sistema padronizado de linguagem. Rev Esc Enferm USP. [periódico on-line]. 2008; [citado 2011 abr 22]; 42(1): [aprox. 8 telas]. Disponível em: http://www.scielo.br. 